\title{
Editorial for economic impact of an enhanced recovery pathway for lung resection
}

\author{
Rajat Kumar, James M. Donahue \\ Division of Cardiothoracic Surgery, University of Alabama at Birmingham School of Medicine, Birmingham, AL, USA \\ Correspondence to: James M. Donahue, MD. Associate Professor of Cardiothoracic Surgery, University of Alabama at Birmingham School of Medicine, \\ 739 Zeigler Research Building, 703 19th Street South, Birmingham, AL 35294, USA. Email: jdonahue@uabmc.edu. \\ Provenance: This is an invited Editorial commissioned by the Section Editor Mathew Thomas (Mayo Clinic, Jacksonville, FL, USA). \\ Comment on: Paci P, Madani A, Lee L, et al. Economic Impact of an Enhanced Recovery Pathway for Lung Resection. Ann Thorac Surg \\ 2017;104:950-7.
}

Submitted Nov 28, 2017. Accepted for publication Dec 14, 2017.

doi: $10.21037 /$ jtd.2017.12.73

View this article at: http://dx.doi.org/10.21037/jtd.2017.12.73

Initiatives to standardize patient care have evolved from fast tracking efforts aimed primarily at reducing costs to more sophisticated enhanced recovery after surgery (ERAS) or enhanced recovery pathways (ERPs). The goals of ERAS pathways are to minimize the perioperative stress response, reduce complications, decrease postoperative length of stay, and enable a faster return to preoperative functional status. Patient care pathways designed to achieve these goals were initially developed in colorectal surgery, and have been validated in studies and in practice (1). More recently, their utility and effectiveness in thoracic surgery have been comparatively assessed (2-8).

In order to be effective, such pathways must address preoperative, intraoperative, and postoperative aspects of care. The preoperative component, which may well be the most critical, centers on patient education. This generally requires a detailed discussion with a team member in clinic and is often summarized with handouts or videos. This step not only serves to inform the patients about their surgery, but more importantly, sets the expectations for their recovery, both in the hospital and after discharge. In particular, for lung cancer patients, these efforts include attention to smoking cessation, optimizing activity before surgery, setting realistic goals for pain management, and establishing the need for patients to participate in early ambulation and pulmonary hygiene efforts in the hospital.

Perioperative and intraoperative aspects of ERAS pathways must involve collaboration and good communication between nursing, anesthesia, and operating room staff in order to facilitate successful implementation of the initiatives. Preemptive analgesia, avoidance of hypothermia, careful fluid management, and reduction of postoperative nausea and vomiting are all factors that play into an individual patient's progress on the pathway. Notably, this discussion often does not include surgical technique, and while minimally invasive approaches contribute to decreased perioperative stress and pain, these pathways have been instituted for patients undergoing both open and minimally invasive surgical procedures.

Postoperative care includes attention to pain control, management of chest tubes, diet, early ambulation, and patient participation in pulmonary hygiene. Pain control strategies include multimodality treatments with nonnarcotics to minimize opioid use, use of epidural or paravertebral catheters, and, more recently, use of longacting liposomal intercostal nerve blocks. Chest tube management is a key component of ERAS pathways in thoracic surgical patients. In addition to being a significant source of pain, chest tube removal is also a major component of discharge time (9). Well documented practices such as use of one chest tube instead of two, chest tube removal with drainage volumes up to $450 \mathrm{~mL} /$ day, and early conversion to a Heimlich valve have been shown to decrease both chest tube duration and length of stay (10).

Despite the enthusiasm for ERAS pathways, evidence for their effectiveness following lung resection has been limited. Most studies comparing outcomes following implementation of such pathways to those prior to 
creation of the pathway have demonstrated a reduction in length of stay by approximately 1 day with no detectable increases in readmission rates $(2,4-6)$. Notably, the only randomized controlled trial of the use of ERAS pathways in thoracic surgery failed to show a decreased length of stay in the ERAS group (3). Although median length of study in this study was long (11 days) in both groups, the lack of change in length of stay in this study does question possible selection biases in the non-randomized studies. In terms of complications, only the randomized study and the current study by Paci et al. show a reduction in pulmonary complications in patients on the ERAS pathway $(3,11)$. Although this finding is encouraging, many of the patients in the current study were included in a previous study from this group and no difference in pulmonary complications was observed in that study (6). Possible reasons for this discrepancy include inclusion patients undergoing thoracoscopic procedures in the current study as well as a reduction in the percentage of patients who are current smokers in the ERAS group in the current study to $35 \%$ from $61 \%$ in the previous study from this group. Adding further disappointment, a recent comparative ERAS study in which all patients underwent a thoracoscopic approach showed no difference in length of stay or complications (8).

Although current results do not lend significant support for ERAS pathways, the intrinsic good sense of these approaches remains very appealing. As the majority of the previously published studies are non-randomized and the randomized study contained a total of only 59 patients, it may well be that these studies are not sufficiently powered to detect differences in complications. In addition, although differences are more pronounced in studies of ERAS pathways in colorectal surgery, the changes implemented in pathways designed for colorectal surgery patients are more novel than those in thoracic surgical studies. Changes instituted in thoracic ERAS studies are centered more on standardization of care as thoracic surgeons have been continually making practice improvements for years. Also, the previous studies may not have allowed enough time for implementation of the changes to become routine at their institutions prior to conducting the studies. As an example, in the current study, adherence to important components of the pathway ranged from only $56-60 \%$.

An important aspect of ERAS pathways that has not been well studied in any surgical field is its effects on costs that are not directly related to the index hospitalization. In the current study, the authors capitalize on data available through the Canadian medical system to rigorously evaluate the effect of ERAS implementation not only on institutional costs, but also on costs to both the health care system in terms of rehabilitation and assisted care and to society in terms of out of pocket expenses and productivity losses from both patients and care givers being out of work. This analysis provides a much fuller picture of what ERAS pathways are intended to do, which is enhancing functional recovery. Looking at just costs for the immediate postoperative hospitalization without exploring the costs related to post-discharge expenditures blunts the true potential benefit of ERAS pathways. Importantly, to be balanced, the authors include costs associated with implementing an ERAS pathway such as hiring a coordinator and printing educational resources. Of the 16 data values presented, 6 of them are statistically significant and all are in favor the ERAS pathway. The most striking cost savings came from societal costs, which were estimated at $\$ 15,799$ for the conventional care group vs. $\$ 11,402$ for the ERAS group. This is a critical finding, as patients need to continue to recover at home after discharge, with the burden of recovery often falling on their family or other members of their support structure. By studying the results of sending patients home in better shape and providing the education and resources necessary for a faster return to normal activities, the true value of ERAS pathways may become more evident.

\section{Acknowledgements}

None.

\section{Footnote}

Conflicts of Interest: The authors have no conflicts of interest to declare.

\section{References}

1. Nicholson A, Lowe MC, Parker J, et al. Systematic review and meta-analysis of enhanced recovery programmes in surgical patients. Br J Surg 2014;101:172-88.

2. Maruyama R, Miyake T, Kojo M, et al. Establishment of a clinical pathway as an effective tool to reduce hospitalization and charges after video-assisted thoracoscopic pulmonary resection. Jpn J Thorac Cardiovasc Surg 2006;54:387-90.

3. Muehling BM, Halter GL, Schelzig H, et al. Reduction of postoperative pulmonary complications after lung surgery 
using a fast track clinical pathway. Eur J Cardiothorac Surg 2008;34:174-80.

4. Salati M, Brunelli A, Xiumè F, et al. Does fast-tracking increase the readmission rate after pulmonary resection? A case-matched study. Eur J Cardiothorac Surg 2012;41:1083-7; discussion 1087.

5. Numan RC, Klomp HM, Li W, et al. A clinical audit in a multidisciplinary care path for thoracic surgery: an instrument for continuous quality improvement. Lung Cancer 2012;78:270-5.

6. Madani A, Fiore JF Jr, Wang Y, et al. An enhanced recovery pathway reduces duration of stay and complications after open pulmonary lobectomy. Surgery 2015;158:899-908; discussion 908-10.

7. Scarci M, Solli P, Bedetti B. Enhanced recovery pathway for thoracic surgery in the UK. J Thorac Dis 2016;8:S78-83.

8. Brunelli A, Thomas C, Dinesh P, et al. Enhanced recovery pathway versus standard care in patients undergoing videoassisted thoracoscopic lobectomy. J Thorac Cardiovasc Surg 2017;154:2084-90.

9. Kwiatt M, Tarbox A, Seamon MJ, et al. Thoracostomy tubes: a comprehensive review of complications and related topics. Int J Crit Illn Inj Sci 2014;4:143-55.

10. Cerfolio RJ, Bryant AS. Results of a prospective algorithm to remove chest tubes after pulmonary resection with high output. J Thorac Cardiovasc Surg 2008;135:269-73.

11. Paci P, Madani A, Lee L, et al. Economic Impact of an Enhanced Recovery Pathway for Lung Resection. Ann Thorac Surg 2017;104:950-7.
Cite this article as: Kumar R, Donahue JM. Editorial for economic impact of an enhanced recovery pathway for lung resection. J Thorac Dis 2018;10(1):7-9. doi: 10.21037/ jtd.2017.12.73 